\title{
MEDIDAS DE FRECUENCIA, ASOCIACIÓN E IMPACTO EN INVESTIGACIÓN APLICADA
}

\author{
J.A. MIRÓN CANELO. \\ M. ALONSO SARDÓN \\ Departamento de Medicina Preventiva y Salud Pública \\ Facultad de Medicina \\ Universidad de Salamanca
}

\section{RESUMEN}

En la Sociedad actual del conocimiento y la información es preciso disponer de herramientas básicas para medir los fenómenos epidemiológicos como la enfermedad, la incapacidad o la siniestralidad laboral. Las formas de medir son instrumentos que se deben conocer y aplicar para planificar y tomar decisiones en Salud Pública y en Salud Laboral.

El objetivo de este artículo es dar a conocer las principales medidas de frecuencia, asociación e impacto utilizadas en investigación aplicada, clínica o de Salud Pública para tratar de medir, valorar y estimar la importancia de los problemas de salud y enfermedades relacionadas con el trabajo, de los factores de riesgo ocupacionales y otros eventos relacionados con la seguridad e higiene en el trabajo y, en definitiva, con la Salud de los trabajadores.

Se describen los conceptos teóricos de las formas de medir en epidemiología, su interpretación y aplicación práctica de los indicadores básicos utilizados en la práctica habitual de los profesionales de las Ciencias de la Salud.

Su utilidad fundamental es poder disponer de una información objetiva, fiable y precisa que permita tomar decisiones adecuadas y pertinentes en relación con la prevención, seguridad laboral, atención y rehabilitación de los trabajadores.

\section{PALABRAS CLAVES}

Medidas de frecuencia, asociación e impacto. Epidemiología. Investigación aplicada.

\section{ABSTRACT}

In the society's current knowledge and information is necessary to have basic tools to measure the epidemic phenomena such as illness, disability or workplace accidents. The ways of measuring are instruments that they must know and apply to plan and take decisions on Public Health and Labour/Occupational Health.

The aim of this article is to inform about the most important measures of frequency, association and impact used in applied research, clinical or Public Health to try to measure, to value and estimate the importance of health problems and diseases related work, The occupational risk factors and other events related to safety and healthy working conditions and ultimately with the Workers' Health.

We describe the theoretical concepts of ways to measure in epidemiology, their interpretation and practical application of basic indicators used in the practice of professionals in the Health Sciences.

Its usefulness is essential to have an objective, reliable and precise information to make sound decisions and allow relevant to the prevention, labour/occupational safety, care and rehabilitation of the workers.

\section{KEY WORDS}

Measures of frequency, association and impact. Epidemiology. Applied research.. 


\section{JUSTIFICACIÓN E IMPORTANCIA}

La epidemiología tiene entre su aplicaciones y fines la valoración y medición de los eventos relacionados con la Salud y la enfermedad. Para cumplir sus fines se vale de los distintos diseños epidemiológicos, éstos han contribuido al conocimiento y control de las enfermedades y exposiciones ocupacionales.

Entre sus aplicaciones básicas está la de medir los eventos epidemiológicos a través de diversas medidas de frecuencia. Medir la frecuencia de las enfermedades u otros fenómenos relacionados con la Salud de los trabajadores es básico para valorar la situación y/o epidemiología de lo que ocurre en la población o en los diversos colectivos sociales. Entre estos colectivos se encuentran los trabajadores dado que el trabajo es actualmente uno de los factores de riesgo más frecuentemente asociado a enfermedad, ya sea ésta o no catalogada como profesional o como enfermedad relacionada con el trabajo.

En el manejo de los distintos estudios o diseños de investigación es evidente la necesidad de contar con diversos indicadores que permitan medir y comparar entre grupos (expuestos y no expuestos, es decir, por ejemplo entre trabajadores -expuestos- y población general -no expuestos-). Las medidas o indicadores caracterizan lo ocurrido u observado, como consecuencia de los factores de riesgo u exposición, como enfermedades profesionales, enfermedades relacionadas con el trabajo, incapacidad, mortalidad o cualquier evento epidemiológico relacionado con la Salud Ocupacional.

\section{MEDIDAS DE FRECUENCIA DE ENFERMEDAD}

\section{Razón, proporción, tasas y riesgo}

La medida más básica que se utiliza para establecer la frecuencia de una enfermedad es el número de casos o frecuencia absoluta. Esta medida es de gran utilidad en planificación sanitaria y laboral para tomar decisiones en relación con la distribución de los recursos y adecuarlos a las necesidades. Sin embargo, es poco útil en investigación, dado que no permite realizar comparaciones lo que la inhabilita para investigar, comparar y establecer conclusiones. Un ejemplo de $\mathrm{n}^{\mathrm{o}}$ absoluto sería el número de trabajadores que sufren accidentes. Con la misma no podemos estimar el riesgo de accidentes, ni establecer el nivel de riesgo en relación a otra empresa o sector.
Por este y otros motivos, las expresiones que se utilizan para medir la frecuencia de enfermedad o evento epidemiológico y, establecer comparaciones, son: la razón, proporción y la tasa.

La razón es un cociente en el que el numerador no está incluido en el denominador. Su rango es de cero a infinito. La proporción es un cociente en el que el numerador está incluido en el denominador. Las proporciones oscilan en un rango entre 0 y 1 y entre 0 y 100 si se expresa en porcentaje. La proporción indica la probabilidad de que un suceso ocurra. La tasa es una forma especial de medir que tiene en cuenta la variable tiempo. Al incluir ésta, las tasas indican la velocidad con que un proceso o evento se produce. En el numerador refleja el número de sucesos que han ocurrido en un período de tiempo determinado de observación. El denominador refleja el total de sujetos y el tiempo en que se ha estado en riesgo de sufrir el suceso cada uno de los sujetos durante el período de observación (personas-tiempo).

El riesgo es un concepto dinámico y probabilístico individual de desarrollar una enfermedad. La diferencia con la tasa, es que ésta se refiere al grupo estudiado y el riesgo al individuo. En el numerador se incluyen la frecuencia absoluta del número de casos del evento investigado y en el denominador en número total al inicio del período. El resultado es un $\mathrm{n}^{\mathrm{o}}$ abstracto sin unidades, adimensional y su valor es siempre inferior a 1 , salvo que se exprese en porcentajes que tendría un valor entre 0 y $100 \%$.

\section{Incidencia y prevalencia}

La frecuencia con que una enfermedad aparece en la población es fundamental para la toma de decisiones profesionales. Existen dos tipos de medidas de frecuencia, que son las más habitualmente utilizadas, la prevalencia y la incidencia. La prevalencia establece la proporción de sujetos que tienen una enfermedad o característica en un momento determinado. Se limita a describir la situación en un momento dado, no lo que ocurrirá. Por su parte, la incidencia mide la aparición de dicha enfermedad o característica en un período de tiempo.

La incidencia nos indica el número de casos nuevos de una enfermedad común/profesional/relacionada con el trabajo que se desarrollan en una población en riesgo durante un período de tiempo. Se entiende por población en riesgo aquella que puede sufrir el evento -enfermedad o accidente-. Existen dos tipos de medidas de incidencia: la inci- 
dencia acumulada (IA) y la tasa de incidencia o densidad de incidencia (DI).

La IA se calcula dividiendo el número de casos nuevos de una enfermedad profesional que aparece en un período de tiempo por el total de la población en riesgo al principio del período. Expresa, por tanto, la probabilidad de que un individuo libre de enfermedad la desarrolle en un período de tiempo determinado, condicionado a que no muera por otra causa en ese mismo período. En la IA se asume que el tiempo de observación es el mismo para cada individuo (cohorte fija). Es decir, todos los individuos entran a la vez y son observados el mismo tiempo (trabajadores de una obra de la construcción). Esto rara vez ocurre en el mundo laboral. Lo más frecuente es que el tiempo de seguimiento u observación sea distinto para cada sujeto/trabajador (cohorte dinámica). Esto se debe a distintas causas: las personas entran en distintos momentos en el estudio, no todos entran a la vez. Las personas del estudio se pueden perder (pérdidas), por muerte, por cambiar de domicilio o por otras circunstancias que no se puedan conocer. Un ejemplo de cohorte dinámica son los trabajadores de la construcción que entran en un obra, las distintas cuadrillas entran en diferentes momentos, la duración de su exposición ocupacional es variable y los motivos por los que salen de la obra pueden ser varios (incapacidad laboral, accidentes, muerte, baja o despido). Para evitar este problema de la variabilidad de la cohorte se utiliza la tasa de incidencia o densidad de incidencia. En esta medida el numerador es el mismo que el la IA, pero el denominador es el sumatorio del tiempo que ha estado cada individuo/trabajador en riesgo de sufrir el evento/enfermedad. Es decir, total de unidades personatiempo de observación en riesgo. En consecuencia, expresa el potencial instantáneo de cambio en el estado de enfermedad por unidad de tiempo, en relación con la población susceptible en ese momento. Por tanto, es una tasa. Su interpretación es colectiva, y representa el potencial de cambio (velocidad) a la que una población sana va enfermando.

$\mathbf{I A}=\mathrm{N}^{\mathrm{o}}$ de nuevos casos en un período determinado/Población riesgo

TI o DI $=\mathrm{N}^{0}$ de casos nuevos en un período determinado/Personas-tiempo de observación

Así, si en el numerador situamos el número de trabajadores que sufren un accidente a lo largo de un período de tiempo, entre la población laboral con riesgo de accidentarse al principio del período de tiempo, estamos midiendo la incidencia acumulada, o sea, la probabilidad individual que tiene el trabajador de desarrollar el fenómeno de interés durante el tiempo que se valora. Como toda probabilidad, su valor oscila entre 0 y $1(0 \%$ y $100 \%$, si se expresa como porcentaje). Mientras que si en el numerador situamos el número de trabajadores accidentados y en el denominador la cantidad total de horas trabajadas (suma de horas trabajadas de cada trabajador), de esta manera estamos midiendo la frecuencia del problema de salud en función del tiempo de exposición y tiene como unidad de medida la del tiempo del denominador y, con ello, estamos calculando la tasa de incidencia. Si se accidentan 8 trabajadores de una empresa de 100 trabajadores que han generado a lo largo de un año 160.000 horas de trabajo, la tasa de accidentes será de 5 por 100.000 h. trabajadas (8/160000 horas). Este resultado es transformable respecto a las unidades; así, si una jornada laboral tiene $8 \mathrm{~h}$., las $160.000 \mathrm{~h}$. se corresponden con 20.000 jornadas, con lo que la tasa de accidentabilidad anterior equivale a decir 4 accidentes por 10.000 jornadas trabajadas $(8 / 20000$ jornadas). Este parámetro nos permite valorar si el tiempo influye en la posibilidad de que un trabajador se accidente (es obvio que, a mayor tiempo trabajado, mayor es la posibilidad de que sufra un accidente).

La elección de una u otra medida de incidencia viene determinada por el objetivo del estudio. Si el objetivo es predecir el riesgo individual de adquirir una enfermedad ocupacional (profesional o no), se deberá estimar el riesgo de que realmente ocurra mediante la IA. Si el objetivo es conocer el poder patógeno de una enfermedad o fuerza de morbilidad se debe utilizar la TI o DI.

La prevalencia es una proporción en que el numerador está formado por número de casos existentes (nuevos y antiguos) y el denominador por el número total de sujetos en riesgo de pasar el numerador, incluyendo los casos. Se trata de un indicador puntual, en un momento dado, que no incluye el factor tiempo. El rango oscila entre 0 y 1 , en porcentajes entre 0 y 100 . La prevalencia establece la carga de una enfermedad o evento en una determinada población. Así, si en una empresa que consta de 100 trabajadores a fecha 1 de enero hay 6 trabajadores dados de baja el día 1 de enero, la prevalencia es del $6 \%(6 / 100)$. Es una medida estática (no mide cambios en el tiempo) de carga de enfermedad en un colectivo. Concluir en un informe laboral que la prevalencia de trabajadores con sindrome de Burnout (estrés laboral) es del 10\%, equivale a decir, que 10 
de cada 100 trabajadores tienen Burnout, independientemente de si les apareció hace una hora que hace un año (casos nuevos y casos antiguos). En ocasiones, sobre todo coloquialmente, se utiliza la denominación de tasa de prevalencia; pero en realidad no lo es.

La relación entre la incidencia y la prevalencia es estrecha, es decir, están fuertemente relacionadas en procesos patológicos o eventos epidemiológicos cuya DI es relativamente estable, es decir, con pocos cambios. La prevalencia incorpora los casos viejos de una enfermedad, lo que implica que cuanto mayor sea la duración media de la enfermedad, a incidencia constante, mayor será la prevalencia. Para enfermedades o eventos epidemiológicos poco dinámicos, es decir, estacionarios, lo que se traduce en que el número de casos nuevos (casos incidentes) y el número de casos existentes (casos prevalentes) en un momento dado son aproximadamente constantes, la prevalencia se puede expresar como el producto de la incidencia por la duración media de la enfermedad. Esto justifica la utilización preferente de casos incidentes, siempre que el estudio lo permita, ya que cuando se comparan dos grupos, una mayor prevalencia en uno de ellos puede ser debido a una mayor duración de la enfermedad y no a una mayor incidencia, que puede ser similar o menor. Si utilizamos casos prevalentes, se puede llegar a la conclusión errónea de que el factor es causa de enfermedad, cuando en realidad lo que hace es aumentar la duración de la misma. En la tabla 1 se reflejan algunas de las diferencias entre incidencia y prevalencia a tener presentes para realizar una mejor interpretación de estas dos medidas fundamentales para cualquier investigación.

\section{Tabla 1.}

\begin{tabular}{|l|l|}
\hline \multicolumn{2}{|c|}{ TABLA 1 } \\
\hline \hline Incidencia & Prevalencia \\
\hline \hline Indica la probabilidad de desarrollar la enfermedad & Indica la probabilidad de que ya se padezca la enfermedad \\
\hline \hline En el numerador deben estar sólo los casos nuevos & En el numerador están todos los casos, nuevos y viejos \\
\hline \hline Para su cálculo se necesita el seguimiento de los individuos & No necesita seguimiento \\
\hline \hline Su valor no depende de la duración de la enfermedad & Su valor depende de la duración de la enfermedad \\
\hline \hline Valora y cuantifica enfermedades agudas & Valora y cuantifica enfermedades crónicas \\
\hline \hline Medida utilizada para investigar y establecer relaciones causales & Medida utilizada para valorar la carga y el coste de una \\
& enfermedad crónica \\
\hline
\end{tabular}

\section{MEDIDAS DE ASOCIACIÓN E IMPACTO}

Las medidas de asociación e impacto cuantifican la relación existente entre variables independientes y dependientes. Una de ellas la exposición o factor de riesgo y otra el efecto o enfermedad. Lo primero es valorar la asociación y que esta no se pueda deber al azar, para ello se calcula si existe asociación estadística a través de la Ji cuadrada de Mantel-Haenszel $\left(\mathrm{X}_{\mathrm{MH}}\right)$. Si existen diferencias entre lo observado y lo esperado, según el valor de la tabla de distribución normal, se dice que hay asociación estadísticamente significativa. El nivel de significación exigido, a nivel internacional, para establecer asociación estadística se fija en el $\mathrm{p} \leq 0,05$. La $p$ se corresponde con la probabilidad de la diferencia en los riesgos sea debida al azar, o lo que es lo mismo, que en realidad no haya diferencias. En consecuencia, se esta- blece si la asociación se debe o no al azar; pero no su magnitud que es lo que interesa para valorar y medir ésta. Para ello se ha de calcular el riesgo del efecto (enfermedad) en los expuestos a una exposición o factor de riesgo y el riesgo en los no expuestos (no exposición o no existencia de factor de riesgo). Para conocer la magnitud cuantitativa de la fuerza de asociación, criterio causal de validez interna de Bradford Hill, se utilizan medidas del efecto e impacto.

\section{MEDIDAS DE EFECTO}

\section{Riesgo Relativo}

El riesgo relativo (RR) estima la frecuencia del efecto en el grupo de expuestos en relación al de no expuestos. Es decir, estima la magnitud del efecto en 
los expuestos a un factor de riesgo en relación con los no expuestos. Indica el número de veces que es más probable que una enfermedad se desarrolle en el grupo expuesto en relación con el grupo no expuesto.

$$
\begin{gathered}
\mathbf{R R}=\text { Incidencia en expuestos /Incidencia en } \\
\text { no expuestos }
\end{gathered}
$$

También se puede expresar en términos de incidencia, siendo la razón entre la incidencia en el grupo de expuestos (Ie) y la incidencia en no expuestos (Io). Por lo que también se denomina Razón de incidencias o de riesgo.

\section{Razón de Incidencias: $\mathrm{RR}=\mathrm{Ie} / \mathrm{Io}$ 0 \\ Razón de Riesgos: $\mathrm{RR}=\mathrm{Re} / \mathrm{Ro}$}

$\mathrm{Su}$ interpretación es sencilla, un valor de 1 indica que no existe relación entre el factor de riesgo (exposición) y la enfermedad (efecto). Si es mayor que 1 indica que existe asociación positiva entre el factor de estudio y la enfermedad. Cuando es menor que 1, indica una asociación negativa, efecto nulo o indiferente. Cuanto más distinto sea el valor del 1, más fuerte es la asociación. Si es mayor de 1, el factor de estudio o exposición es un factor de riesgo y si es menor que 1 , la exposición protege. Por ejemplo, un valor del RR de 2 indica que la exposición dobla el riesgo, es decir, aumenta un $100 \%$ la probabilidad del efecto (enfermedad).

El RR obtenido es una estimación puntual, por lo que debe calcularse su intervalo de confianza (IC). Si el intervalo de confianza no incluye el valor 1, existe asociación estadísticamente significativa entre el factor de riesgo y el efecto. La ventaja del IC 95\%, frente a la significación a través de $p$, es que permite la inferencia y generalización de los resultados observados a la población de referencia, asumiendo que se ha estudiado a una muestra representativa y aleatoria. Al interpretarlo hay que tener presente la posibilidad de errores no debidos al azar (sesgos o errores sistemáticos).

El RR se debe utilizar como parámetro en aquellos estudios que tengan por objetivo determinar la magnitud de la asociación en términos relativos, es decir en estudios prospectivos como el estudio cohortes y el ensayo clínico (Ver figura 1).

Figura 1. Estructura básica de un diseño de Cohortes.

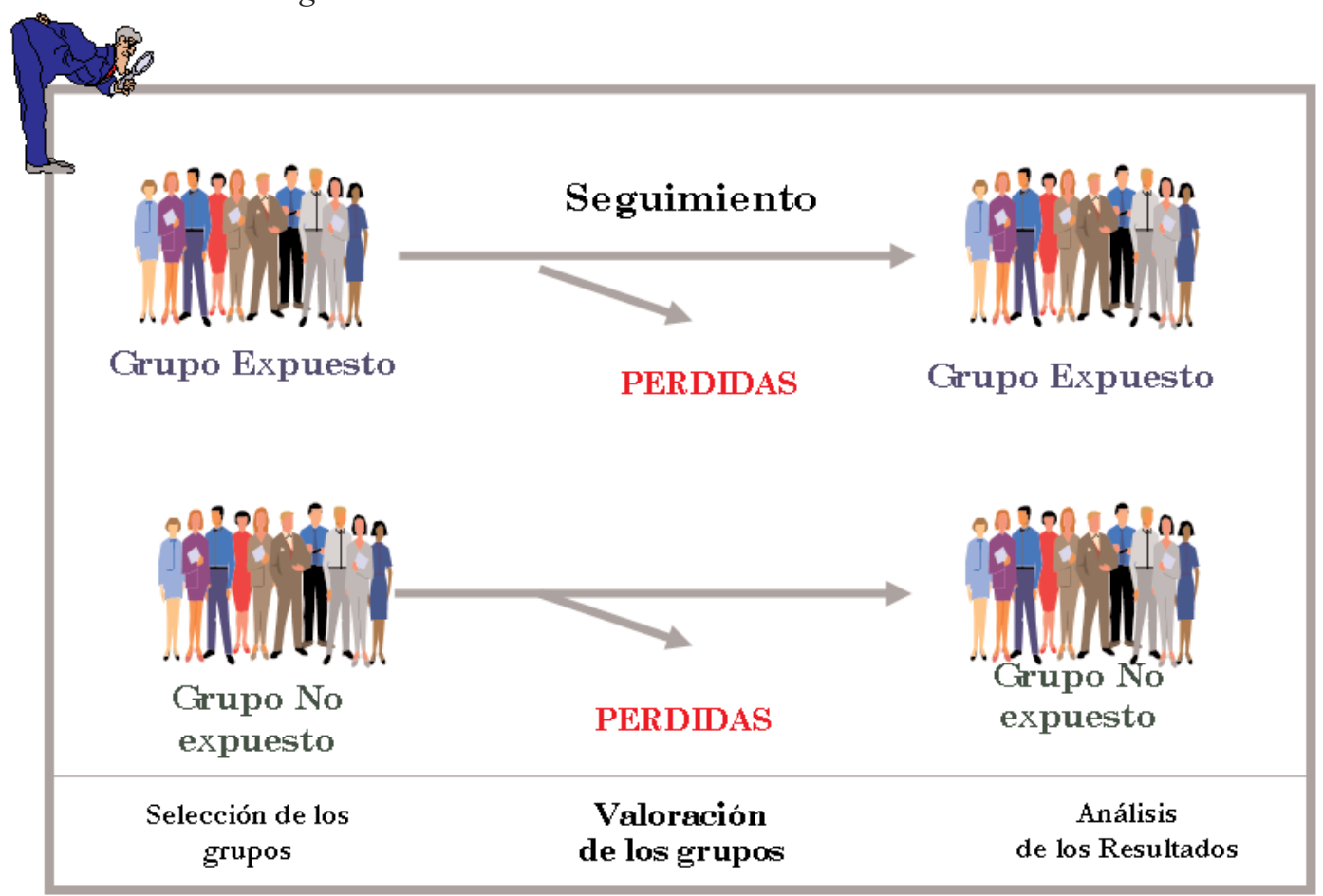




\section{Odds Ratio}

En estudios de cohortes el RR se estima de forma directa ya que se conoce la incidencia de la enfermedad en expuestos y en no expuestos. Por el contrario, en los estudios de casos y controles no se puede calcular la incidencia, porque la población de estudio se selecciona a partir de individuos que ya han desarrollado el efecto, la enfermedad. Así en los estudio de casos y controles se calcula la razón de ventajas o razón de posibilidades, razón de odds u odds ratio (OR) (Ver figura 2). Por Odds se entiende la razón entre la probabilidad de que un suceso ocurra y la probabilidad de que no ocurra. La OR no es más que la razón entre la odds de exposición observada en el grupo de casos $(\mathrm{a} / \mathrm{c})$ y odds de exposición en el grupo control $(b / c)$.

$$
\mathrm{OR}=\mathrm{a} \times \mathrm{d} / \mathrm{b} \times \mathrm{c}
$$

Figura 2. Estructura básica de un diseño de Casos y Controles.

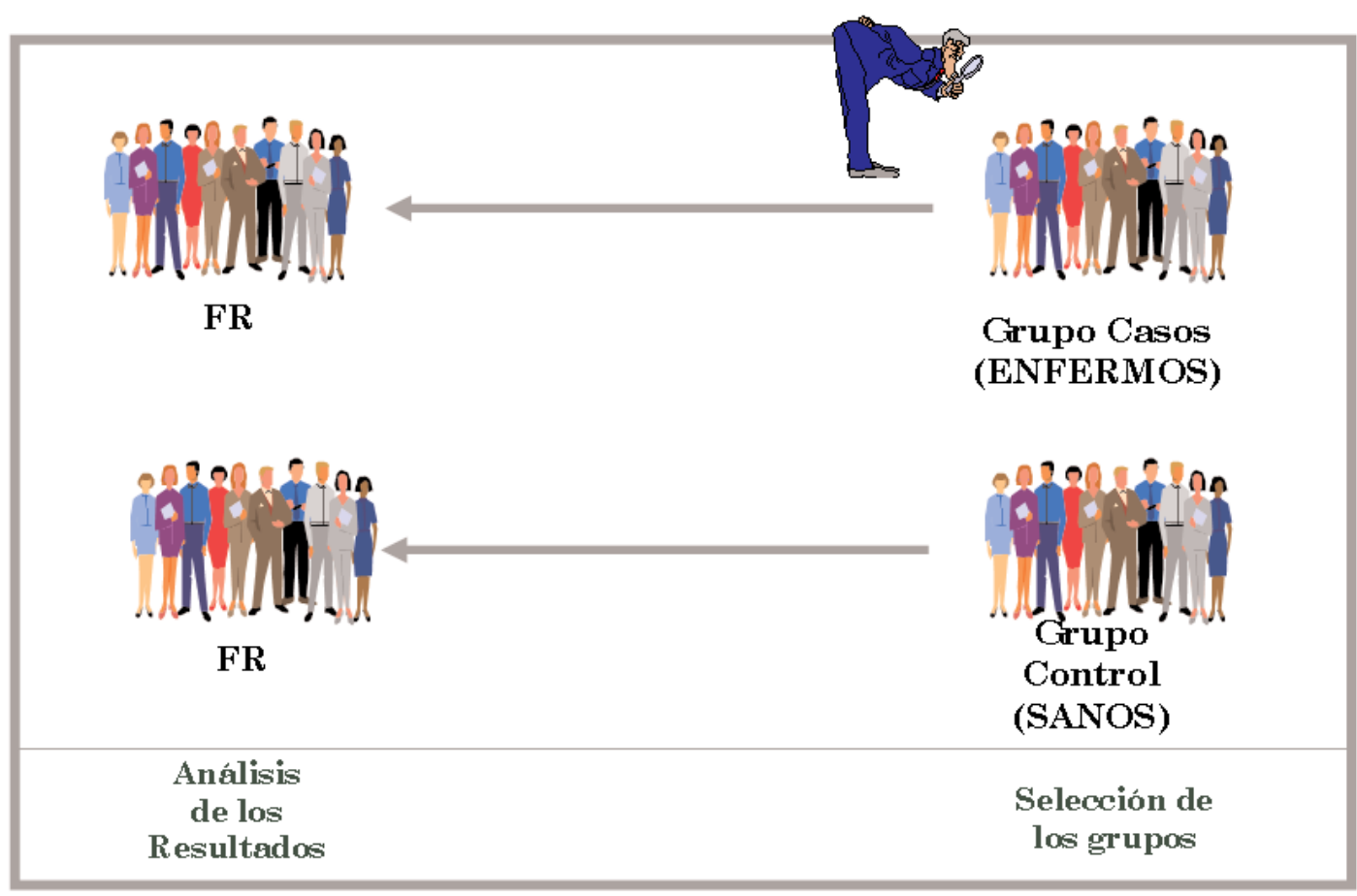

La OR, es actualmente el estimador más utilizado, cada vez se utiliza más como medida de la magnitud de un efecto o asociación, tanto en los estudios de casos y controles, cohortes y ensayos clínicos, como en revisiones sistemáticas y meta-análisis. Se interpreta como equivalente del RR, aunque en realidad la OR sólo es una buena aproximación del RR en determinadas condiciones. Si el riesgo basal (frecuencia del resultado en la población de estudio) es bajo, menor al $10 \%$, ambas medidas son equivalentes. Cuanto mayor es el riesgo basal, la OR exagera la magnitud del efecto en relación con el RR, es decir, más lo sobrestima cuando es mayor que 1 . Por otra parte, la OR es el parámetro más interesante y más fácil de interpretar que proporcionan los análisis multivariantes de regresión logística. En consecuencia, cuanto más alta es la OR, mayor es la fuerza de la asociación entre la enfermedad ocupacional y el determinante (factor de riesgo o exposición ocupacional).

\section{Riesgo Atribuible (RAe) o Diferencia de Riesgo en los expuestos (DRe)}

Es el riesgo de tener el efecto en los expuestos que se debe a la exposición. Se calcula restando al riesgo de los expuestos el de los no expuestos. En consecuencia, sería el riesgo adicional producido por la exposición al factor de riesgo. O lo que es lo mismo, la parte del riesgo individual que se debe al factor de estudio.

$$
\mathrm{RA}=\mathrm{Ie}-\mathrm{Io} \quad \text { ó } \quad \mathrm{DRe}=\mathrm{Re}-\mathrm{Ro}
$$


$\mathrm{Su}$ interpretación cuantitativa y numérica es la siguiente: Valor $>$ que 0 , el factor es de riesgo, $=0$ efecto nulo $\mathrm{y}<$ que 0 factor protector

\section{MEDIDAS DE IMPACTO}

\section{Fracción Etiológica o Atribuible en los expuestos (FEe o RAe)}

Es la proporción de efectos producidos por la exposición en los expuestos. Se calcula dividiendo el riesgo atribuible a la exposición en los expuestos (la DRe) por el total del riesgo en los expuestos.

$$
\begin{gathered}
\mathrm{FEe}=\mathrm{Ie}-\mathrm{Io} / \mathrm{Ie} \\
\mathrm{FAe}=\mathrm{RAe} \text { o DRe/Ie o Re }
\end{gathered}
$$

Indica también la magnitud de la asociación, tanto mayor cuanto más se aproxima a 1 o al $100 \%$ cuando se expresa en porcentajes.

El valor de la FEe o RAe depende exclusivamente del valor del RR, ya que la ecuación siguiente es equivalente a la anterior. Si la expresión $\mathrm{FEe}=\mathrm{Ie}-$ Io/Ie la dividimos por la incidencia en no expuestos (Io). Resulta lo siguiente: $\mathrm{FEe}=\mathrm{RR}-1 / \mathrm{RR}$

En estudios de cohortes, sería $\mathrm{FE}=(\mathrm{RR}-1) / \mathrm{RR}$

En casos y controles, sería $\mathrm{FEe}=(\mathrm{OR}-1) / \mathrm{OR}$.

\section{Diferencia de Riesgo en el total de la población (DRp)}

El significado es equivalente a la DRe; pero referido a la población total. Es por tanto, el riesgo producido por la exposición en la población total.

$$
\mathrm{DRp}=\mathrm{It}-\mathrm{Io}=\mathrm{Ip}-\mathrm{Io}
$$

\section{Fracción Etiológica o Atribuible en el total de la población (FEp o FAp)}

El significado es equivalente a la FEe; pero referido al total de efectos. Es por tanto, la proporción del total de los efectos producidos por la exposición. También se puede valorar como la proporción de enfermedad (efectos) que desaparecería en el conjunto de la población (de la incidencia total It o poblacional Ip), si eliminamos la exposición.

Su cálculo se realiza, según la siguiente fórmula:

$$
\mathrm{FEp}=\mathrm{Ip}-\mathrm{Io} / \mathrm{Ip}
$$

Es un parámetro importante pues indica la repercusión de una causa en el número total de casos de toda la población. Si se comparan las FAp para diferentes causas o variables, aquella que obtenga la más elevada es la más importante.

En la tabla 2 pueden observarse las fórmulas de

\begin{tabular}{|c|c|c|}
\hline \multicolumn{3}{|c|}{ TABLA 2} \\
\hline Media & Estimación & IC $95 \%$ \\
\hline $\mathrm{RR}$ & Ie / Io & $R R^{1 \pm \frac{Z_{\alpha-1 / 2^{*}}}{\sqrt{\chi_{M-H}^{2}}}}$ \\
\hline OR & $\mathrm{a} \times \mathrm{d} / \mathrm{b} \times \mathrm{c}$ & $O R R^{1 \pm \frac{Z_{\alpha-1 / 2^{*}}}{\sqrt{\chi_{M-H}^{2}}}}$ \\
\hline DRe o RAe & $\mathrm{Re}-\mathrm{Ro}$ o Ie - Io & $R A e\left(1 \pm \frac{Z_{\alpha-1 / 2^{*}}}{\sqrt{\chi_{M-H}^{2}}}\right)$ \\
\hline FAe o FEe & $\begin{array}{c}\mathrm{Ie}-\mathrm{Io} / \mathrm{Ie} \\
(\mathrm{RR}-1) / \mathrm{RR}\end{array}$ & $1-(1-F E e)^{1 \pm \frac{Z_{\alpha-1 / 2 *}}{\sqrt{\chi_{M-H}^{2}}}}$ \\
\hline DRp o RAp & It - Io & $R A p\left(1 \pm \frac{Z_{\alpha-1 / 2^{*}}}{\sqrt{\chi_{M-H}^{2}}}\right)$ \\
\hline FAp o FEp & It - Io / It & $1-(1-F E p)^{1 \pm \frac{Z_{\alpha-1 / 2^{*}}}{\sqrt{\chi_{M-H}^{2}}}}$ \\
\hline
\end{tabular}
calcular las estimaciones puntuales y los intervalos de confianza de las distintas medidas de asociación e impacto.

(*) $\mathrm{Z}_{\alpha-1 / 2}=1,96$, para un intervalo de confianza del $96 \%$. 


\section{EJERCICIO PRÁCTICO}

Antes de la disponibilidad generalizada de la vacuna correspondiente, un riesgo conocido de los trabajadores sanitarios era la infección por el virus de la hepatitis-B (VHB). A partir de los datos de la tabla 3, comparamos el riesgo de infección en los profesionales sanitarios frente al experimentado por otro tipo de personal.

Tabla 3

\begin{tabular}{|c|c|c|c|}
\hline & \multicolumn{2}{|c|}{ EFECTO } & \\
\hline EXPOSICIÓN & $\begin{array}{c}\mathbf{E}+ \\
\text { Infectado por VHB }\end{array}$ & $\begin{array}{c}\text { E- } \\
\text { No infectado VHB }\end{array}$ & \\
\hline $\begin{array}{l}\text { FR+ } \\
\text { Profesionales sanitarios }\end{array}$ & a & b & $\mathrm{n}_{1}$ \\
\hline $\begin{array}{l}\text { FR- } \\
\text { Resto del personal }\end{array}$ & c & d & $\mathbf{n}_{0}$ \\
\hline & $\mathrm{m}_{1}$ & $\mathrm{~m}_{0}$ & $\mathbf{N}_{5000}$ \\
\hline
\end{tabular}

Así, podemos calcular:

- El riesgo de infección en los profesionales sanitarios (incidencia en expuestos) sería de $0,01\left(\mathrm{Ie}=\mathrm{a} / \mathrm{n}_{1}=40 / 4000\right)$.

- El riesgo de infección del resto del personal (incidencia en no expuestos) sería de 0,002 $\left(\right.$ Io $\left.=\mathrm{c} / \mathrm{n}_{0}=2 / 1000\right)$.

A la vista de los resultados, está claro que el riesgo de infección por VHB es mayor en los profesionales sanitarios que en el resto del personal. Pero es necesario cuantificar esta diferencia mediante una magnitud: el RIESGO RELATIVO (RR), que consiste en el cociente del riesgo en expuestos (en este caso, los profesionales sanitarios), entre el riesgo en no expuestos (el resto del personal):

$$
\mathrm{RR}=\mathrm{Ie} / \mathrm{Io}=0,01 / 0,002=5
$$

El RR indica el número de veces que ocurre el suceso de interés en el grupo de expuestos frente al de no expuestos; el valor 5 obtenido representa que la frecuencia de infección por VHB es 5 veces mayor en el grupo de profesionales sanitarios que en el resto del personal.

El RR mide la fuerza de asociación; esta asociación será tanto más fuerte cuanto más difiera de 1, el valor del RR. Cuando el RR toma valores superiores a 1, la asociación es positiva (el factor de riesgo en estudio favorece la aparición de la enfermedad). Por el contrario, cuando el RR toma valores inferiores a 1, la asociación es negativa (en estos casos el factor de riesgo en estudio puede ser considerado como protector). En el caso en el que el RR es igual a 1, no hay asociación entre la exposición y la enfermedad.

Otra medida de asociación que se puede calcular es la ODDS RATIO (OR) o razón de probabilidades (cociente entre una probabilidad y su complementaria $=p / 1-p)$. Así, podemos calcular:

- La odds del efecto entre los expuestos, sabiendo que la probabilidad de infección por VHB entre los expuestos (profesionales sanitarios) es 40/4000 y que, por lo tanto, el valor de su complementario es 3960/4000.

Odds de $V H B$ en profesionales sanitarios $=$ $40 / 4000 / 3960 / 4000=40 / 3960$

- La odds del efecto en los no expuestos, sabiendo que la probabilidad de infección por VHB entre los no expuestos (resto del personal) es 2/1000 y que, por lo tanto, el valor de su complementario es 998/1000.

Odds de $V H B$ en el resto del personal $=$ $2 / 1000 / 998 / 1000=2 / 998$

La OR será el cociente entre ambas odds: 
$\mathrm{OR}=40 / 3960 / 2 / 998=40 \times 998 / 3960 \times 2=$ $=\mathrm{a} \times \mathrm{d} / \mathrm{b} \times \mathrm{c}=5,04 \mathrm{a}$

El valor de la OR obtenido se interpreta de manera similar al RR.

También podemos calcular la diferencia de riesgos o RIESGO ATRIBUIBLE en expuestos (RAe), que mide el exceso de riesgo de infección por VHB que presentan los profesionales sanitarios (grupo expuesto) en relación al resto del personal, como consecuencia de la exposición.

$\mathrm{RAe}=\mathrm{Ie}-\mathrm{Io}=0,01-0,002=0,008$

Y el RIESGO ATRIBUIBLE en la población (RAp), que en este caso mide el exceso de riesgo de infección por VHB que presentan toda la población (trabajadores sanitarios), expuesta y no expuesta (profesionales sanitarios y resto del personal), como consecuencia de la presencia del factor.

$$
\text { RAp }=\text { It }- \text { Io }=0,0084-0,002=0,0064
$$

Donde It (incidencia total) representa riesgo de infección por VHB en la población (It $=\mathrm{m}_{1} / \mathrm{N}=$ 42/5000).
Por último, para cuantificar la repercusión que tendría en la población y entre los expuestos la modificación o supresión del factor de riesgo de estudio, calculamos las correspondientes fracciones etiológicas o atribuibles:

FRACCIÓN ETIOLÓGICA O ATRIBUIBLE en expuestos o porcentaje de riesgo atribuible en expuestos, cuyo valor sería $80 \%$, que corresponde a la proporción de infección por VHB entre los profesionales sanitarios (expuestos) que se debe a la exposición; o dicho en términos de beneficio, si eliminamos la exposición entre los profesionales sanitarios (expuestos), reducimos en un $80 \%$ la infección por VHB.

$\mathrm{FEe}=\mathrm{Ie}-\mathrm{Io} / \mathrm{Ie}=\mathrm{RAe} / \mathrm{Ie}=0,008 / 0,01=0,80$

FRACCIÓN ETIOLÓGICA O ATRIBUIBLE en la población o porcentaje de riesgo atribuible en la población, cuyo valor sería $76 \%$, que en este caso corresponde a la proporción de infección por VHB en la población (profesionales sanitarios y resto del personal) que se debe a la exposición; o dicho en términos de beneficio, si eliminamos la exposición en la población (profesionales sanitarios y resto del personal), reducimos en un $76 \%$ la infección por VHB.

$\mathrm{FEe}=\mathrm{It}-\mathrm{Io} / \mathrm{It}=\mathrm{RAp} / \mathrm{Ie}=0,0064 / 0,0084=0,76$ 


\section{BIBLIOGRAFÍA}

Abraira V. Medidas del efecto de un tratamiento (I): reducción absoluta del riesgo, reducción relativa del riesgo y riesgo relativo. SEMERGEN. 2000; 26b (11): 535-6.

Abraira V. Medidas del efecto de un tratamiento (II): odds ratio y número necesario para tratar. SEMERGEN. 2001; 27 (8): 418-20.

Abraira V. Medidas de asociación en la investigación clínica: aplicación a un estudio hipotético de asociación entre hipertensión y consumo de sal. Clin Invest Arterioscler. 1994; 6: 190-3.

Aino H, Yanagisawa S, Kamae I. The number needed to treat needs an associated odds estimation. J Public Health. 2004; 26 (1):84-7.

Argimón Pallás JM, Jimenez Villa J. Métodos de investigación clínica y epidemiológica. Barcelona: Elservier, 2006.

Caycoya M, Mirón Canelo JA. Cáncer de pulmón en Asturias. Un estudio de casos y controles. Gac Sanit (Barc) 2003; 17: 226-230.
Jaeschke R, Guyatt G, Shannon H, Walter S, Cook D, Heddle N. Assessing the effects of treatment: measures of association. Can Med Assoc J. 1995; 152 (6): 351-7.

Martínez-González MA, Irala-estevez J, GuillenGrima F. ¿Qué es la odds ratio?. Med Clín (Barc) 1999; 112:416-422.

Rothman K.J. Epidemiología Moderna. Madrid: Díaz de Santos, 1987.

Schiaffino A, Rodríguez M, Pasarín MI, Regidor E, Borrell, Fernández E. ¿Odds ratio o razón de proporciones? Su utilización en estudios transversales. Gac Sant 2003; 17: 70-74.

Tapia Granados JA. Incidencia: concepto, terminología y análisis dimensional. Med Clín (Barc) 1994; 103: 140-142.

Villar Álvarez F, Banegas Banegas JR, González Enriquez J, Martín Moreno JM, Rodríguez Artalejo F. Diseño y análisis epidemiológico. Rev Rol Enf 1987; 112: 29-3 